\title{
Microbial Metabolite Production for Accelerated Metal and Radionuclide Bioremediation
}

(U)

September 21, 2004

C. E. Turick

Y.G. Kritzas

Westinghouse Savannah River Company

Savannah River Site

Aiken, SC 29801 
This document was prepared in conjunction with work accomplished under Contract No. DE-AC09-96SR18500 with the U. S. Department of Energy.

\section{DISCLAIMER}

This report was prepared as an account of work sponsored by an agency of the United States Government. Neither the United States Government nor any agency thereof, nor any of their employees, makes any warranty, express or implied, or assumes any legal liability or responsibility for the accuracy, completeness, or usefulness of any information, apparatus, product or process disclosed, or represents that its use would not infringe privately owned rights. Reference herein to any specific commercial product, process or service by trade name, trademark, manufacturer, or otherwise does not necessarily constitute or imply its endorsement, recommendation, or favoring by the United States Government or any agency thereof. The views and opinions of authors expressed herein do not necessarily state or reflect those of the United States Government or any agency thereof.

This report has been reproduced directly from the best available copy.

Available for sale to the public, in paper, from: U.S. Department of Commerce, National Technical Information Service, 5285 Port Royal Road, Springfield, VA 22161, phone: (800) 553-6847, fax: (703) 605-6900

email: orders@ntis.fedworld.gov

online ordering: http://www.ntis.gov/help/index.asp

Available electronically at http://www.osti.gov/bridge

Available for a processing fee to U.S. Department of Energy and its contractors, in paper, from: U.S. Department of Energy, Office of Scientific and Technical Information, P.O. Box 62, Oak Ridge, TN 37831-0062,

phone: (865)576-8401,

fax: (865)576-5728

email: $\underline{\text { reports@ adonis.osti.gov }}$ 


\section{Microbial Metabolite Production for Accelerated Metal and Radionuclide Bioremediation}

\subsection{EXECUTIVE SUMMARY}

Biogeochemical activity is an ongoing and dynamic process due to bacterial activity in the subsurface. Bacteria contribute significantly to biotransformation of metals and radionuclides. As basic science reveals more information about specific mechanisms of bacterial-metal reduction, an even greater contribution of bacteria to biogeochemical activities is realized. An understanding and application of the mechanisms of metal and radionuclide reduction offers tremendous potential for development into bioremedial processes and technologies.

Most bacteria are capable of biogeochemical transformation as a result of meeting nutrient requirements. These assimilatory mechanisms for metals transformation include production of small molecules that serve as electron shuttles for metal reduction. This contribution to biogeochemistry is small however due to only trace requirements for minerals by bacteria.

Dissimilatory metal reducing bacteria (DMRB) reduce oxidized metals and insoluble mineral oxides as a means for biological energy production during growth. These types of bacteria offer considerable potential for bioremediation of environments contaminated with toxic metals and radionuclides because of the relatively large amount of metal biotransformation they require for growth. One of the mechanisms employed by some DMRB for electron transfer to insoluble metal oxides is melanin production. The electrochemical properties of melanin provide this polymeric, humic-type compound with electron shuttling properties. Melanin, specifically, pyomelanin, increases the rate and degree of metal reduction in DMRB as a function of pyomelanin concentration. Due to its electron shuttling behavior, only low femtogram quantities per cell are required to significantly increase metal reduction capacity of DMRB.

Melanin production is not limited to DMRB. In fact melanin is one of the most common pigments produced by biological systems. Numerous soil microorganisms produce melanin, contributing to about 2-4\% of the humic fraction of soils (Ellis and Griffiths, 1997). Our recent work has shown that melanin production by one species of bacteria could be used by other species for metal reduction. This melanin "sharing" is the area of focus for this project. In addition, melanin contributes to significant increases in metal reduction by both assimilatory and dissimilatory metal reducing bacteria.

Stimulation of melanin production in the subsurface offers potential for accelerating metal reduction. Another focus of this project was to determine the potential of melanin production in portions of the Tims Branch watershed as it relates to metal and radionuclide immobilization insitu. 


\section{TABLE OF CONTENTS}

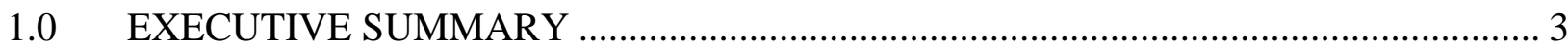

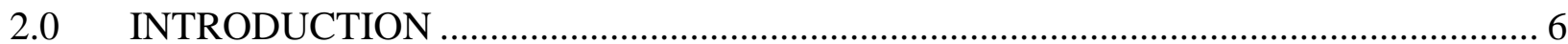

3.0 MATERIALS AND METHODS ......................................................................... 7

3.1 QUANTIFICATION OF MELANIN PRODUCING MICRORGANISMS AT TIMS

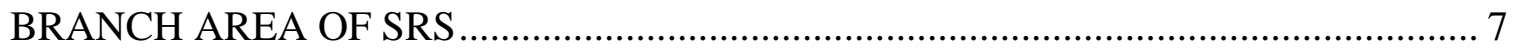

Site Selection ........................................................................................... 7

3.1.2 Most probable Number (MPN) assay for melanin production. ................................ 7

3.2 CHARACTERIZATION OF MELANIN-LIKE PIGMENTS FROM SRS SOILS ......... 7

3.2.1 Isolation of Melanin-Type Pigment-Producing Microorganisms ............................ 7

3.2.2 Characterization of Melanin-Type Microbial Pigments ........................................... 7

P.3 PYOMELANIN UTILIZATION STUDIES ........................................................ 8

3.3.1 Microbial Interspecies Pyomelanin Utilization ............................................... 8

3.3.2 Quantitative Pyomelanin Sorption.................................................................. 8

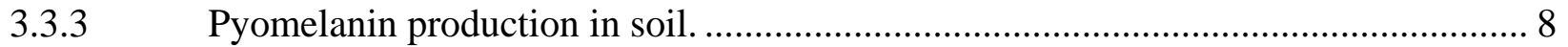

Effects of aeration on pyomelanin production..................................................... 9

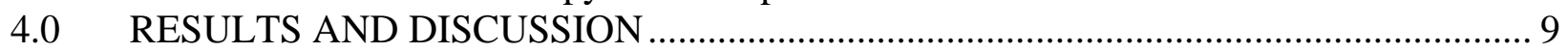

4.1 QUANTIFICATION OF MELANIN PRODUCING MICROORGANISMS AT TIMS

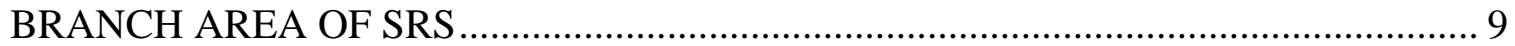

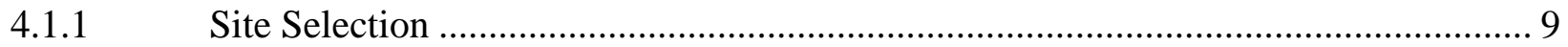

4.1.2 Most probable Number (MPN) assay for melanin production ................................ 9

4.2 CHARACTERIZATION OF MELANIN-LIKE PIGMENTS FROM SRS SOILS .......... 9

4.2.1 Isolation of Melanin-Type Pigment-Producing Microorganisms ............................ 9

4.2.2 Characterization of Melanin-Type Microbial Pigments ......................................... 10

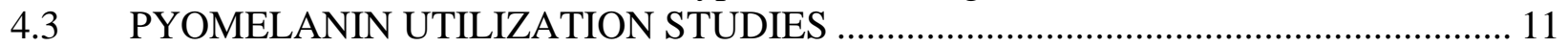

4.3.1 Microbial Interspecies Pyomelanin Utilization ................................................... 11

4.3.2 Quantitative Pyomelanin Sorption ................................................................ 11

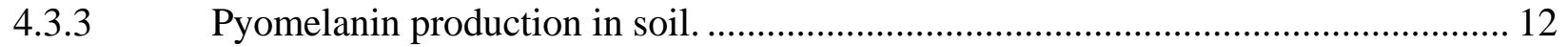

4.3.4 Effects of aeration on pyomelanin production................................................. 13

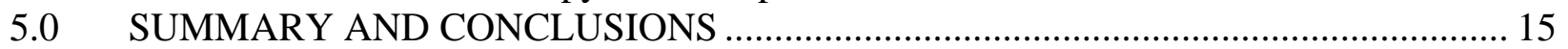

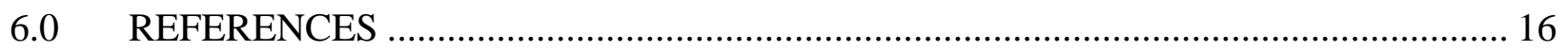




\section{LIST OF FIGURES}

Figure 1. Growth of pyomelanin producing isolate from Tims Branch .....................................10

Figure 2. Melanin production with and without sulcotrione ...............................................10

Figure 3. Pyomelanin-assisted iron mineral reduction .....................................................11

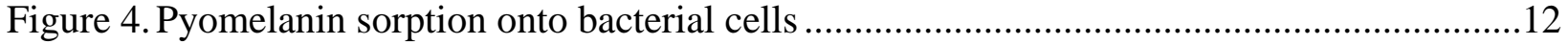

Figure 5. Effects of tyrosine with and without supplemental lactate to soils..............................13

Figure 6. Pyomelanin production in liquid media over time...................................................14 


\subsection{INTRODUCTION}

Microorganisms play significant roles in geological processes and mineral cycling through the formation and transformation of minerals (Ehrlich, 1996). Bacterial reduction of soluble metals ultimately results in metal immobilization and mineral formation (Ehrlich, 1996). Metal and radionuclide reduction is coupled to energy conservation and growth by dissimilatory metal reducing bacteria (DMRB) when metals are used as terminal electron acceptors (Lovley 1991, 1993).

Accelerated reduction of metals has also been demonstrated via bacterial reduction of humictype compounds. The humics accelerate metal reduction by acting as conduits for electron flow from bacteria to metals and radionuclides. Some examples of humic-type compounds include anthroquinone disulfonate and humic acids (Lovley et al. 1996) as well as pyomelanin (Turick 2001; Turick et al. 2002 ; Turick et al. 2003).

Humic compounds are recalcitrant, heterogeneous polymers that contain polyaromatic structures with quinones and hydroquinones as well as carboxyl and hydroxyl groups (Scott and Martin, 1990). The quinones associated with humics have redox-cycling properties, which allows the same humic molecule to be used repeatedly for electron transfer. Humics originate from the partial decomposition of organic molecules such as tannins (Scott and Martin, 1990). Microbial genesis of soil humics is primarily through organic matter decomposition and provides an input of "new humics," which react better with metals than do aged humics (Wu and Tanoue, 2001). In addition a portion of naturally occurring soil humic compounds is the result of microbial melanin-pigment production (Scott and Martin, 1990; Bell and Wheeler, 1986).

The presence of environmentally occurring humics is advantageous to bioremedial efforts because they can accelerate bacterial metal and radionuclide reduction. However the quantity and redox cycling qualities will vary considerably from site to site (Scott et al., 1998) and therefore cannot be relied upon to expedite cleanup efforts at all DOE facilities. The addition of humics to the contaminated subsurface are expected to accelerate remedial efforts, but prohibitive costs and difficulty in delivering these mineral-sorptive compounds to their desired location could make humic supplementation highly impractical.

The approach of the work described here is to determine the feasibility of in-situ production of melanin by metal reducing microorganisms for the purpose of accelerated in-situ metal reduction. Bacteria in the genus Shewanella couple the reduction of metals for energy production and growth. In addition, several species produce the humic-type compound pyomelanin, which substantially increases metal reduction rates by these bacteria. This study was conducted to determine the feasibility of stimulating pyomelanin production in Tims Branch soils for the purpose of accelerated metal reduction in-situ through; 1) quantification of melanin producing microorganisms, 2) characterizing melanin produced in these soils, 3) determining the pyomelanin sorption and metal reduction capacity of a broad range of common soil bacteria. 


\subsection{MATERIALS AND METHODS}

\subsection{QUANTIFICATION OF MELANIN PRODUCING MICRORGANISMS AT TIMS BRANCH AREA OF SRS}

\subsubsection{Site Selection and soil collection}

The Tims Branch watershed makes up a portion of SRS and parts of this watershed have been impacted in the past with U(VI) and Ni. Uncontaminated soils representative of this watershed were selected for this study. In addition sampling and research activities were coordinated with other researchers at the Savannah River Ecology Lab (SREL). Soil samples were collected aseptically and immediately stored on ice until delivery to the lab. Soil samples were then refrigerated until processing.

\subsubsection{Most probable Number (MPN) assay for melanin production.}

Soil dilutions from $10^{-2}-10^{-8}$ were prepared in lactate basal salt medium supplemented with $1 \mathrm{~g} / \mathrm{l}$ tyrosine. Controls received the same treatment except tyrosine was omitted. Each tube of the 3 tube MPN contained $10 \mathrm{mls}$ of growth medium and soil. Tubes were incubated for 4 weeks at $25^{\circ} \mathrm{C}$ and shaken at $100 \mathrm{rpm}$. Increased pigmentation as a result of tyrosine amendments was determined spectrophotometrically by scanning the supernatant fluid of each test tube from 600$300 \mathrm{~nm}$. Tubes with increased OD in this range, relative to controls were marked positive for pigment production from tyrosine. The number of positive tubes per dilution were used to calculate the most probable number of cells.

\subsection{CHARACTERIZATION OF MELANIN-LIKE PIGMENTS FROM SRS SOILS}

\subsubsection{Isolation of Melanin-Type Pigment-Producing Microorganisms}

To isolate pigment-producing microorganisms, samples were removed aseptically from the soil MPNs above and inoculated onto tryptic soy agar supplemented with tyrosine (2g/l) (TSAT), using the spread plate method. Following incubation at $20^{\circ} \mathrm{C}$ for 1 week, colonies demonstrating pigmentation were transferred from TSAT to a lactate basal salts agar with $2 \mathrm{~g} / \mathrm{l}$ tyrosine and monitored for pigmentation.

\subsubsection{Characterization of Melanin-Type Microbial Pigments}

The chemical sulcotrione is a specific competitive inhibitor of the enzyme 4-hydroxyphenyl pyruvate dioxygenase (4HPPD), which is the enzyme required for pyomelanin production. Methods were developed using sulcotrione to determine if pigment production is a result of 4HPPD. Pure cultures of soil isolates were grown in defined minimal media that contained $6 \mathrm{~g} / \mathrm{l}$ lactate as the carbon and energy source. Tyrosine was supplemented to the defined medium to promote pyomelanin production. Sulcotrione $(18 \mu \mathrm{M})$ was added prior to pigmentation. Pigment production was monitored in cell free culture fluid and visually. 


\subsection{PYOMELANIN UTILIZATION STUDIES}

\subsubsection{Microbial Interspecies Pyomelanin Utilization}

Pyomelanin was produced in batch culture and concentrated through acidification and centrifugation as previously described (Turick et al. 2002). Concentrated pyomelanin was then dialyzed at $4^{\circ} \mathrm{C}$ with deionized water. The water was changed periodically until the $\mathrm{pH}$ was sustained at 6-7. After dialysis, the pyomelanin was dried at $60^{\circ}$ over steam. To produce a stock solution, dried pyomelanin was dissolved into $0.1 \mathrm{~N} \mathrm{NaOH}, \mathrm{pH}$ adjusted to 7 with $\mathrm{HCl}$, autoclaved and stored at $4^{\circ}$ for future use.

To determine the effects of pyomelanin contact with various microorganisms, the following pure cultures of bacteria were used, Shewanella oneidensis, Escherichi coli, Bacillus cereus, Pseudomonas putida, P. sryngae and Micrococcus luteus. These organisms represent a broad phylogenetic representation of environmental bacteria. Cultures were grown for $24 \mathrm{~h}$ in $25 \mathrm{mls}$ TSB at $25^{\circ} \mathrm{C}$, shaken at $100 \mathrm{rpm}$. Cultures were then harvested and concentrated through centrifugation $(8000 \mathrm{xg}$ ) and washed twice with $25 \mathrm{mM}$ sodium bicarbonate buffer. Aliquots were then transferred to test tubes containing $25 \mathrm{mM}$, anaerobic sodium bicarbonate buffer and $50 \mu \mathrm{l}$ hydrous ferric oxide (HFO). Pyomelanin as added from stock to a final concentration of $0.1 \mu \mathrm{g} / \mathrm{ml}$ to the test tubes. All additions were done under anaerobic conditions $\left(90 \% \mathrm{~N}_{2} /\right.$ $10 \% \mathrm{CO}_{2}$ ) and the test tubes were sealed air tight with viton stoppers. Controls did not receive pyomelanin. Hydrogen $(100 \mathrm{kPa})$ was added to each test tube. Hydrogen was used as the electron donor in these studies. Incubation was at $28^{\circ} \mathrm{C}$, at $100 \mathrm{rpm}$, for 6 days. Pyomelanin reduction by bacteria was determined by the ability of bacteria to reduce HFO to soluble Fe(II). $\mathrm{Fe}(\mathrm{II})$ was quantified with the ferrozine assay (Turick et al. 2002).

\subsubsection{Quantitative Pyomelanin Sorption}

Quantitative assessment of pyomelanin sorption was determined with the bacterial cultures from the study above. After 6 days incubation, $1 \mathrm{ml}$ samples were taken from each test tube (above), centrifuged ( $8000 \mathrm{x}$ g) and washed 2 times with $25 \mathrm{mM}$ sodium bicarbonate. The cell pellet was then assayed for pyomelanin. Quantification of pyomelanin was determined with the nitro-blue tetrazolium (NBT) assay described previously (Paz et al, 1991; Turick et al. 2003). This assay is specific for quinones, a chemical component of pyomelanin.

\subsubsection{Pyomelanin production in soil.}

Melanin production was determined directly in Tims Branch soil samples. One gram of soil was added to $25 \mathrm{mls}$ basal salts medium supplemented with $1 \mathrm{~g} / \mathrm{l}$ tyrosine. A portion of the samples received supplemental lactate ( $9 \mathrm{mls} 60 \% \mathrm{Na}$ lactate syrup/ $\mathrm{l}$ ). Controls received no carbon source. Soils were incubated in $250 \mathrm{ml}$ flasks with loose covers to allow for aeration. Incubation was at $28^{\circ} \mathrm{C}$ for 1 month, shaken at $100 \mathrm{rpm}$. After one month sample supernatant fluids were assayed as above for increased OD relative to controls. 


\subsubsection{Effects of aeration on pyomelanin production.}

Soil samples were prepared as in section 3.3.3 but were incubated statically, not shaken. Flasks containing the cultures were sealed air tight to limit aeration.

\subsection{RESULTS AND DISCUSSION}

\subsection{QUANTIFICATION OF MELANIN PRODUCING MICROORGANISMS AT TIMS BRANCH AREA OF SRS}

\subsubsection{Site Selection}

Soils sampled were in the Pond 25 area and were obtained near the Tims Branch creek (Sample A) as well as soils at higher elevation, away from the creek (Sample B). Soil samples were collected aseptically and immediately stored on ice until delivery to the lab. Soil samples were then refrigerated until processing.

\subsubsection{Most probable Number (MPN) assay for melanin production}

Bacterial densities of pigment producers for both soil samples from Tims Branch demonstrated MPN values of $2.9 \times 10^{7}$ cells /g wet wt of soil with a $95 \%$ confidence limit of $1.1 \times 10^{8}$ to $7.8 \times 10^{6}$. Pigmentation occurred more rapidly in the higher dilutions and may have been a result of inhibitory components in the soils at the lower dilutions or a result of nutrient competition. The degree of pigmentation was similar with all positive tubes irregardless of dilution after 1 month incubation indicating that any inhibition that may have occurred early in the incubation did not persist.

\subsection{CHARACTERIZATION OF MELANIN-LIKE PIGMENTS FROM SRS SOILS}

\subsubsection{Isolation of Melanin-Type Pigment-Producing Microorganisms}

Bacteria were isolated from the highest positive MPN tubes (above) to confirm melanin production on solid media. Two pure cultures were isolated and were determined to produce melanin on defined media with tyrosine. Media without tyrosine did not result in pigment production. 


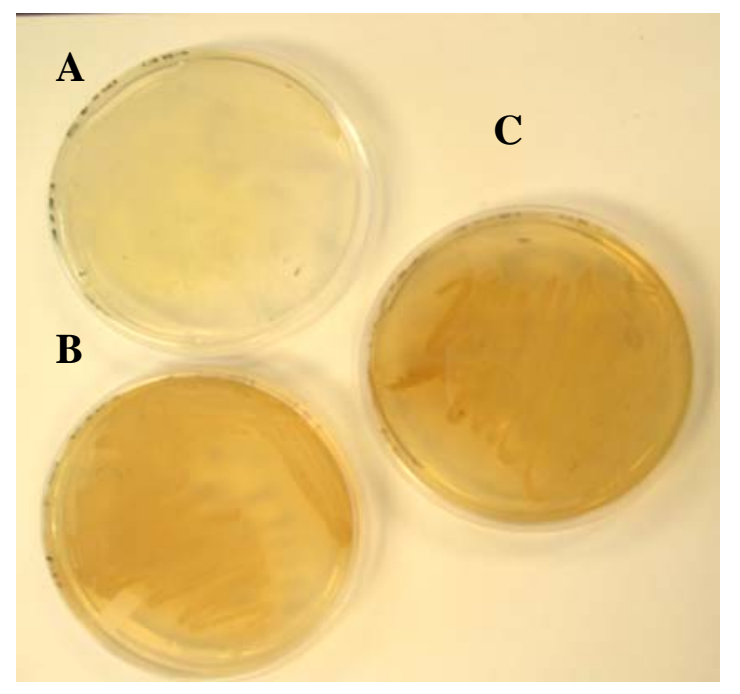

Figure 1. Growth of pyomelanin producing isolate from Tims Branch. Results after 1 week growth on LBSM without tyrosine (A) and with tyrosine (B). Redish-brown coloration is a result of pyomelanin production from tyrosine. More intense pyomelanin production was evident after 2 weeks growth on the same medium (C).

\subsubsection{Characterization of Melanin-Type Microbial Pigments}

Melanin producing isolates were unable to produce melanin in the presence of the enzyme inhibitor sulcotrione. This enzyme inhibitor is specific for the enzyme responsible for pyomelanin production. It was then concluded that pyomelanin is the type of melanin produced by these isolates. Because these isolates represent the most common pigment producers of our samples, the potential for pyomelanin production at this sight is expected to be significant.
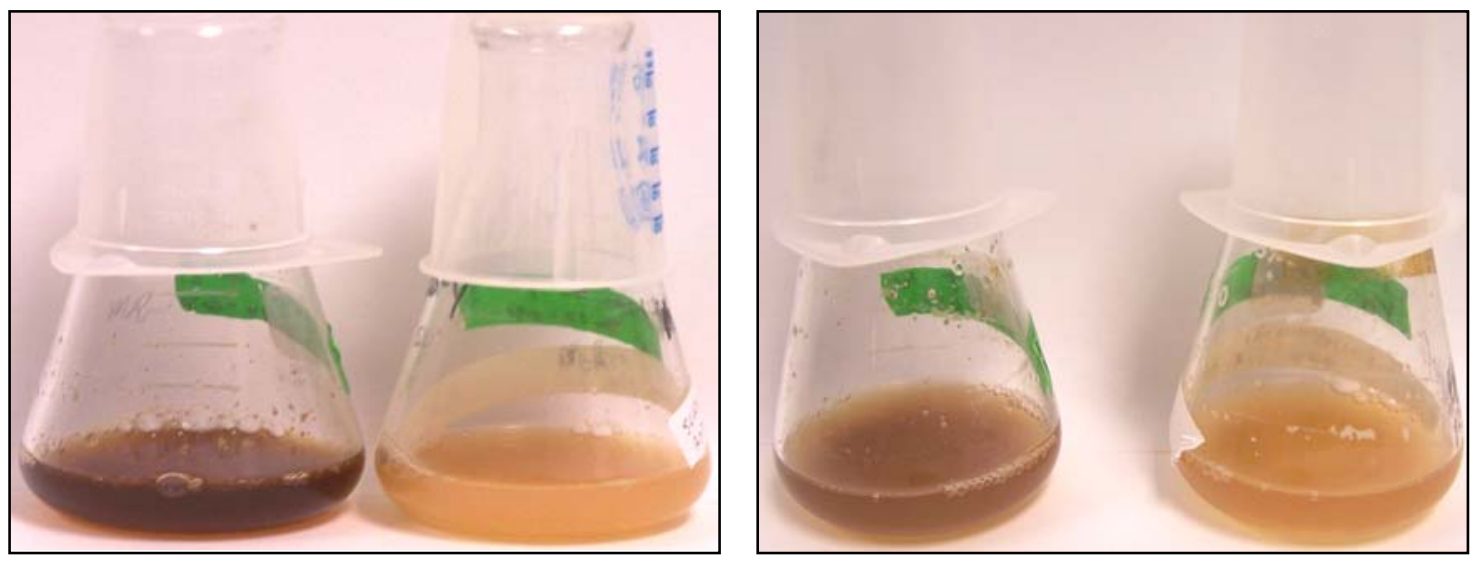

Figure 2. Melanin production with and without sulcotrione. Melanin production ceased upon addition of the pyomelanin inhibitor sulcotrione (flasks on right of each picture) when tested on the 2 most common pigment producers isolated from the Tims Branch area. Flasks on left of each picture did not receive inhibitor treatment. 


\subsection{PYOMELANIN UTILIZATION STUDIES}

\subsubsection{Microbial Interspecies Pyomelanin Utilization}

All bacteria assayed were able to reduce the insoluble iron oxide HFO to a significantly greater degree in the presence of purified pyomelanin than without it (Fig. 3). In this study hydrogen served as the electron donor. During this process the bacterial cultures were capable of hydrogen oxidation to electrons and protons. The resulting reduction of HFO in the presence of hydrogen therefore demonstrates the capacity of these bacteria to couple hydrogen oxidation to pyomelanin reduction and ultimately melanin oxidation to HFO reduction. By serving as an electron shuttle, a molecule of pyomelanin can be reused numerous times. This can potentially provide a considerable degree of metal reduction potential to soil microbes associated with pyomelanin.

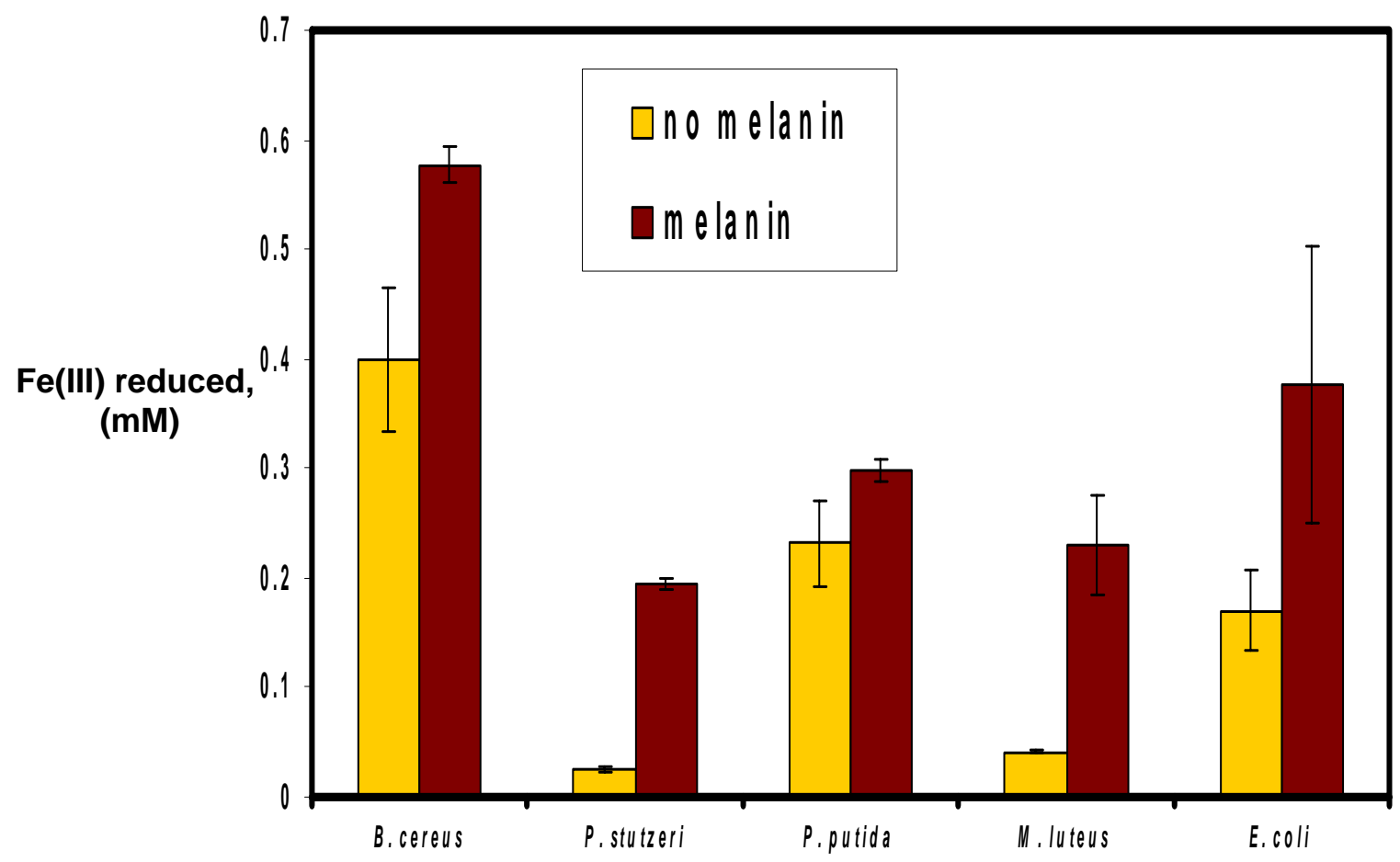

Figure 3. Pyomelanin-assisted iron mineral reduction. Addition of $0.1 \mu \mathrm{g} / \mathrm{ml}$ of pyomelanin to bacterial cultures with hydrous ferric oxide resulted in an increase in the amount of Fe(II) evolved after 1 week of incubation.

\subsubsection{Quantitative Pyomelanin Sorption}

Results of the NBT assay demonstrated that pyomelanin adsorption occurred with all bacterial types analyzed (Fig 4). Since surface associated pyomelanin results in significant increases in metal reduction (Turick et al. 2003), sorption of pyomelanin by a broad range of bacteria, even at low femtogram levels per cell, suggests that pyomelanin overproduction in the subsurface by some bacteria will enlist a large portion of the microbial community into metal reduction. 


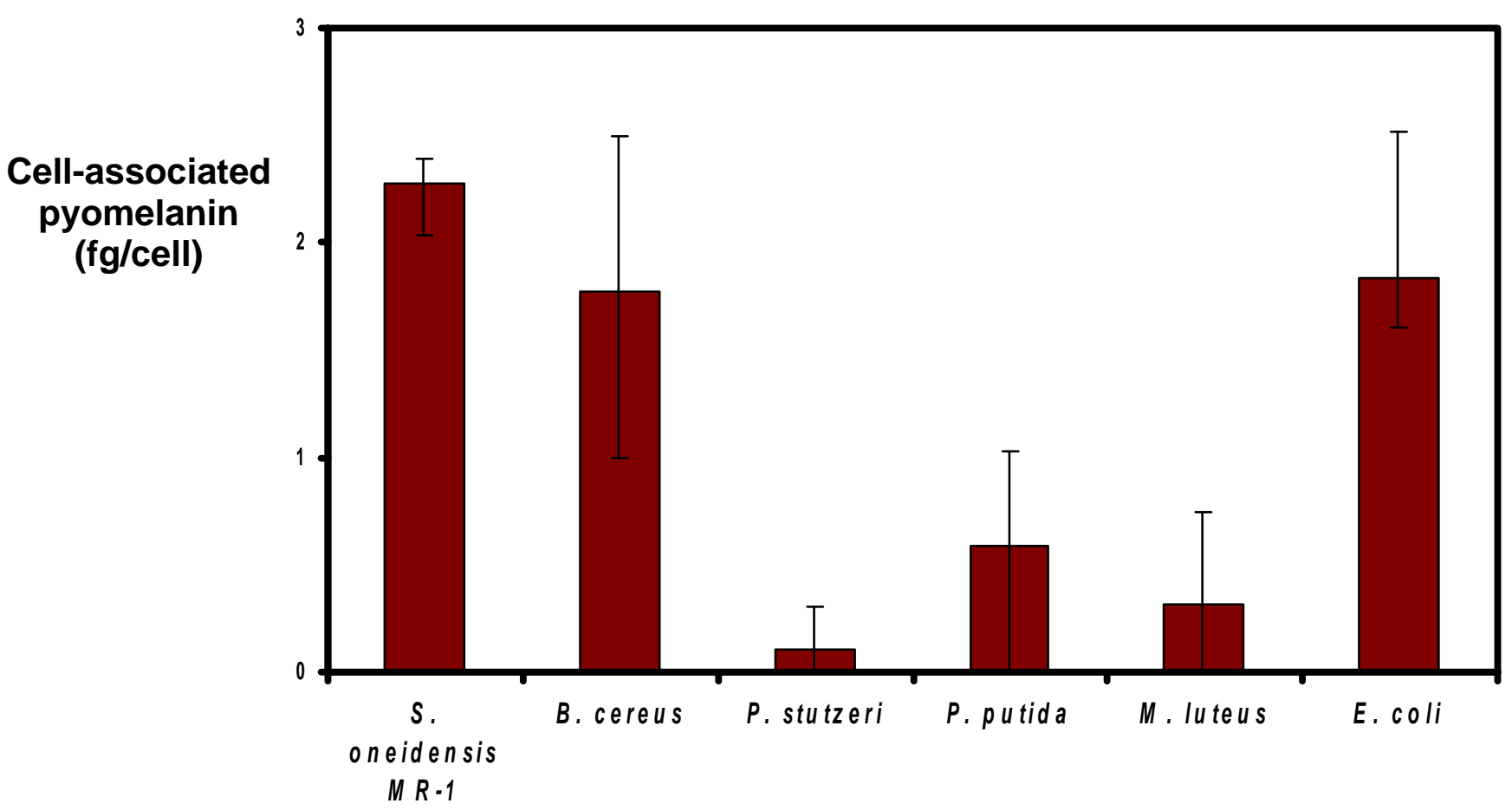

Figure 4. Pyomelanin sorption onto bacterial cells. After 1 week incubation all bacterial types tested demonstrated an ability to sorb pyomelanin to the cell surface.

\subsubsection{Pyomelanin production in soil.}

Addition of tyrosine to all soils tested from Tims Branch resulted in increased pigmentation relative to controls. When tyrosine was supplemented with lactate as a carbon source, the degree of melanin production increased (Fig. 5). The most common type of pigmentation produced by bacterial isolates from this study was determined to be pyomelanin and the increased OD in this study is most likely attributable to increased pyomelanin production. 


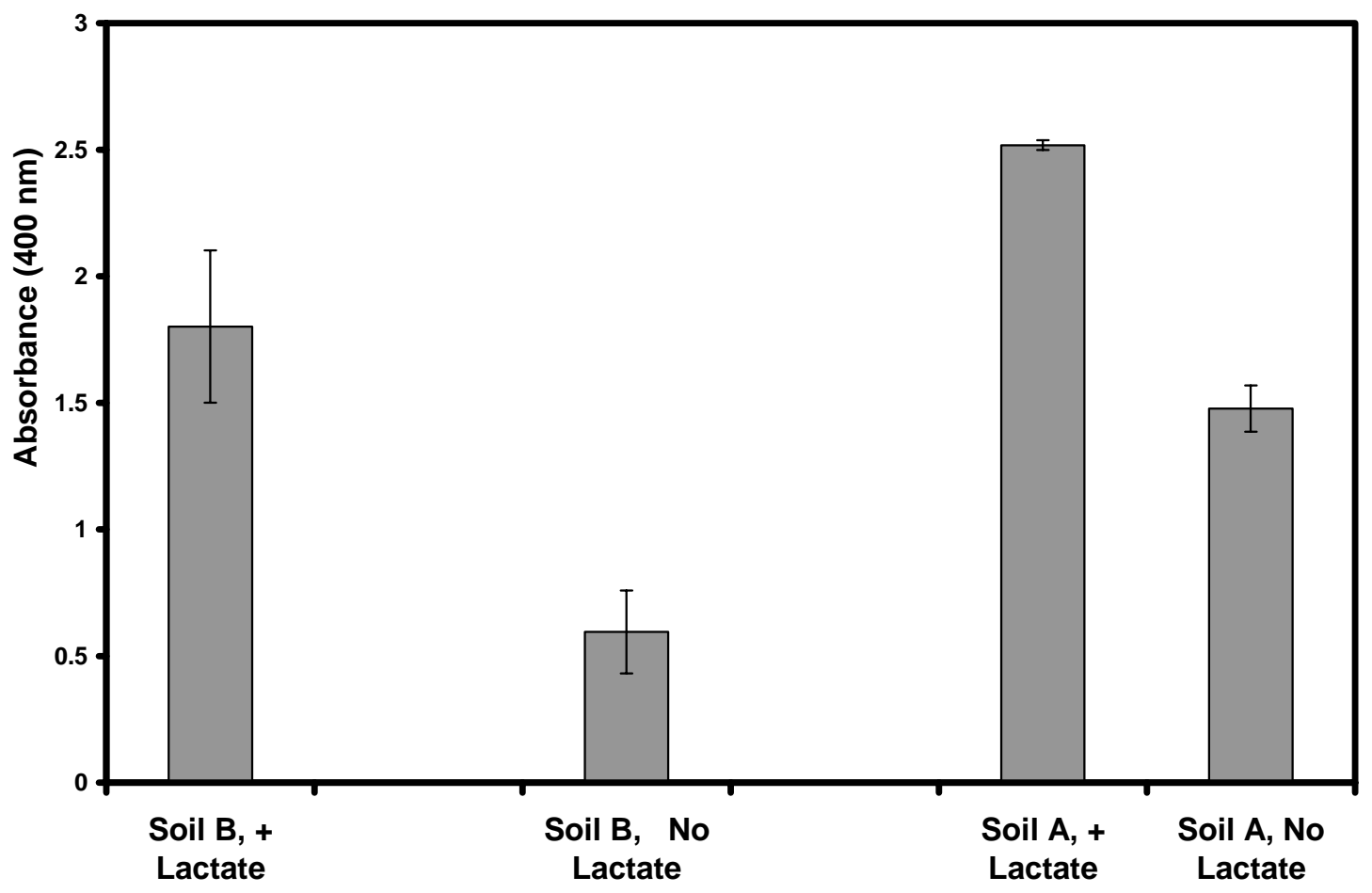

Figure 5. Effects of tyrosine with and without supplemental lactate to soils. Increased pigmentation (as increased $\mathrm{OD}_{400 \mathrm{~nm}}$ ) resulted after addition of tyrosine to all soils tested (controls subtracted). A greater increase in pigmentation of the culture liquid resulted with supplemental additions of lactate.

\subsubsection{Effects of aeration on pyomelanin production}

Pyomelanin production in well aerated cultures (shaken at $100 \mathrm{rpm}$ ) was not significantly different than cultures grown statically (not shaken or stirred) (data not shown). This demonstrated that melanin production is not dependent on high oxygen tension. The production of pyomelanin in sealed test tubes demonstrated that pyomelanin production appears to begin at the highest oxygen levels (top of liquid) (Fig. 6). Over time pyomelanin production increased down the test tube, indicating that pyomelanin production in the subsurface will impact the upper most portions of the surface prior to movement to more anaerobic zones. The culture conditions during growth would have kept oxygen levels low due to bacterial growth. Pyomelanin production appears to be a result of oxygen stress and likely occurs under very low oxygen concentrations. 


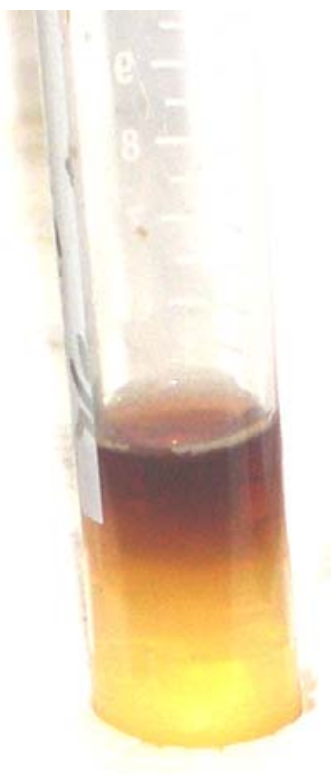

1 week

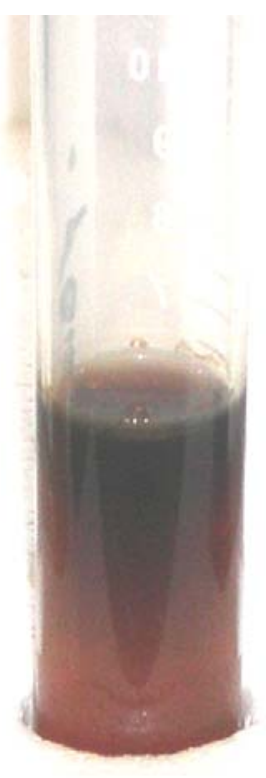

2 weeks

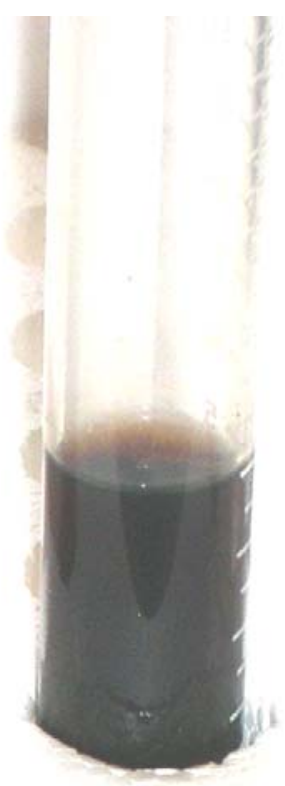

3 weeks

Figure 6. Pyomelanin production in liquid media over time. Pyomelanin production from a pure culture of $S$. oneidensis $\mathrm{hmgA}^{-}$demonstrated increased pyomelanin production over time with increased darkening of the growth medium as pyomelanin production intensified.

Production of pyomelanin began at the uppermost levels of the culture, indicating a requirement for at least trace amounts of oxygen. 


\subsection{SUMMARY AND CONCLUSIONS}

\subsection{DETERMINATION OF BACTERIAL PIGMENT PRODUCTION}

Contamination from metals and radionuclides exists throughout the DOE system including SRS. Remediation technologies exist for treating these contaminants but when metals and radionuclides are present in low concentrations these technologies may cease to be cost effective. This project addresses research to support environmental restoration and stabilization needs. The ultimate goal of this work is to develop an environmental biotechnology based application for accelerated metal and radionuclide reduction and in-situ stabilization.

The main focus of this work is on microbial melanin production in the Tims Branch area of SRS. Melanin has redox cycling properties that increases the rate of electron transfer from the cell surface to soluble and insoluble metals. Previous work in this area has focused on a particular type of melanin, pyomelanin. Pyomelanin is produced from the catabolism of the amino acids tyrosine and/or phenylalanine. Pyomelanin production and metal reduction rates increase as a function of tyrosine or phenylalanine concentration. The overall goal of this FY04 work was to demonstrate that increased production of pyomelanin through tyrosine addition increases the rates of metal and radionuclide reduction, leading to their in-situ stabilization.

Pyomelanin production was tentatively determined in all soils tested from Tims Branch by the production of redish brown pigment in tyrosine supplemented growth studies. Bacterial isolates from these soils were capable of pigment production similar to pyomelanin when grown with tyrosine. The pigments produced were diffusible in solid growth media but bacterial cultures also demonstrated darker coloration in the presence of these pigments, indicating melanin sorption under aerobic conditions. Pyomelanin producing microbes were quantified from these soils and demonstrated a density of as much as $10^{7}$ cells/g soil.

Pyomelanin sorption to bacterial surfaces was demonstrated with various strains of bacteria that do not produce melanin and are not dissimilatory metal reducers. Insoluble Fe(III)-oxides were reduced to $\mathrm{Fe}(\mathrm{II})$ to a significant degree by all bacteria tested after pyomelanin sorption to their surfaces.

This study has demonstrated the ubiquity of melanin production at Tims Branch soils and the capacity for the melanin to sorb onto a wide variety of bacteria, enabling them to reduce soluble and insoluble metal in-situ. Tyrosine (or phenylalanine) amendments to soil along with lactate result in melanin production in Tims Branch. The melanin produced enlists numerous bacterial species into metal reduction in-situ and thereby accelerates the rate of metal immobilization. 


\subsection{REFERENCES}

Ehrlich, H.L. 1996. In. Geomicrobiology. pp.312-367. Marcel Decker Inc. N.Y., N.Y.

Bell, A.A. and M.H. Wheeler. 1986. Annual Review of Phytopathology. 24:411-451.

Ellis, D.H. and D.A. Griffiths. 1997. Can. J. Microbiol.

20:1379-1386.

Lovley, D. R. 1991. Dissimilatory Fe(III) and Mn(IV) reduction. Microbiol. Rev. 55:259-287.

Lovley, D. R. 1993. Dissimilatory metal reduction. Ann. Rev. Microbiol. 47:263-290.

Lovley, D.R., J.D. Coates, E.L. Blunt-Harris, E.J.P. Phillips, and J.C. Woodward. 1996. Nature. 382:445-448.

Paz, M.A. R.A. Fluckiger, A. Boak, H.M. Kagan, P.M. Gallop. 1991. J. Biol. Chem. 266:689692.

Scott, D.E. and J.P. Martin. 1990. Chapter 3. In Humic Substances in Soil and Crop Sciences: Selected Readings, edited by P. MacCarthy, C.E. Clapp, R.L. Malcolm, and P.R. Bloom. Madison, WI: Soil Science Society of America, Inc.

Scott, D.T., D.M. McKnight, E. L. Blunt-Harris, S.E. Kolesar and D.R. Lovley. 1998.

Environmental Science and Technology. 32:2984-2989.

Turick, C.E., 2001.The physiological role and characterization of melanin produced by Shewanella algae BrY. Doctoral Dissertation. Durham, N.C.: University of New Hampshire.

Turick, C.E., L.S. Tisa, and F. Caccavo Jr. 2002. Applied and Environmental Microbiology 68:2436-2444.

Turick, C.E., L.S. Tisa, and F. Caccavo Jr. 2003. FEMS Microbiol. Lett. 220:99-104

Wu, F., and E. Tanoue. 2001. Environ. Sci. Technol.35;3646-3652, 